\title{
A NOTE ON FREE SUBSEMIGROUPS WITH TWO GENERATORS ${ }^{1}$
}

\author{
BY E. K. BLUM
}

\author{
Communicated by J. W. Green, March 10, 1965
}

Let $\Sigma$ be an alphabet and $F(\Sigma)$ the free semigroup of words over $\Sigma$ [3]. Let $E=\left\{w_{1}, w_{2}\right\} \subset F(\Sigma)$, where $w_{1}$ and $w_{2}$ are nonempty words. We give a necessary and sufficient condition on $E$ in order that the subsemigroup generated by $E$ be free with $E$ as its unique irreducible generating set.

DEFINITION. If $w, z \in F(\Sigma)$ are such that $w=z^{n}, n \geqq 0$, then $z$ is a root of $w$. The root of $w$ having minimum word length (maximum $n$ ) is the primitive root of $w$ and is denoted by $\rho(w)$.

REMARK. It follows from results in [2], [3], [4] that $\rho\left(w^{k}\right)=\rho(w)$ for any $k>0$. Also, if $w_{1} w_{2}=w_{2} w_{1}$ and $w_{1}, w_{2}$ are both nonempty words, then $\rho\left(w_{1}\right)=\rho\left(w_{2}\right)$. The converse is obviously true; i.e. if $\rho\left(w_{1}\right)=\rho\left(w_{2}\right)$, then $w_{1} w_{2}=w_{2} w_{1}$.

TheOREM. Let $E=\left\{w_{1}, w_{2}\right\}$ be a set of two nonempty words in $F(\Sigma)$. The subsemigroup generated by $E$ is free with $E$ as its unique irreducible generating set if and only if $\rho\left(w_{1}\right) \neq \rho\left(w_{2}\right)$.

Proof. Suppose $\rho\left(w_{1}\right)=\rho\left(w_{2}\right)=z$; then by the remark above, $w_{1} w_{2}$ $=w_{2} w_{1}$ is a nontrivial relation which implies that the subsemigroup generated by $E$ is not a free semigroup with $E$ as its unique irreducible generating set.

Now suppose that the subsemigroup generated by $E$ is not free with $E$ as its unique irreducible generating set. Then there exists a nontrivial relation of the form

$$
w_{1}^{n_{1}} w_{2}^{n_{2}} \cdots=w_{2}^{m_{1}} w_{1}^{m_{2}} \cdots \text {, }
$$

with $n_{i}>0, m_{i}>0$. Without loss of generality, we may assume that the length of $w_{2}$ is not greater than the length of $w_{1}$. This together with (1) implies that $w_{2}$ is a left-factor of $w_{1}$. Hence, we have

$$
w_{1}=w_{2}^{q} t_{0},
$$

where $q \geqq 1$ is taken to be such that $w_{2}^{q}$ is the largest power of $w_{2}$ which is a left-factor of $w_{1}$. Substituting in (1), we obtain $\left(w_{2}^{t} t_{0}\right) w_{2} \ldots$ $=w_{2}^{m_{1}}\left(w_{2}^{2} t_{0}\right) \cdots$,

1 This work was partially supported by NSF grant GP 2993. 


$$
t_{0} w_{2} \cdots=w_{2}^{m_{1}} t_{0} \cdots \text {. }
$$

By the definition of $q, w_{2}$ cannot be a left-factor of $t_{0}$. Therefore, by (3), $t_{0}$ must be a left-factor of $w_{2}$. We may write $w_{2}=t_{0} t_{1}$.

Equation (1) clearly has the form $v w_{1}=v^{\prime} w_{2}$, where $v, v^{\prime} \in F(\Sigma)$. This implies that $w_{2}$ is a right-factor of $w_{1}$. However, $w_{1}=w_{2}^{g-1} t_{0} t_{1} t_{0}$. Since the length of $t_{1} t_{0}$ is equal to the length of $w_{2}$, it follows that $w_{2}=t_{1} t_{0}$. Thus, $t_{1} t_{0}=t_{0} t_{1}$ and by the remark, $\rho\left(t_{0}\right)=\rho\left(t_{1}\right)=z$. This implies $\rho\left(w_{2}\right)=z$ and by (2) we also have $\rho\left(w_{1}\right)=z$. This completes the proof.

\title{
REFERENCES
}

1. P. M. Cohn, On subsemigroups of free semigroups, Proc. Amer. Math. Soc. 3 (1962), 347-351.

2. M. W. Curtis, On some commutative sets in a free monoid, Abstract 64T-203, Notices Amer. Math. Soc. 11 (1964), 250.

3. E. S. Lyapin, Semigroups, Translations of Mathematical Monographs, Vol. 3, Amer. Math. Soc., Providence, R. I., 1963.

4. R. C. Lyndon and M. P. Schutzenberger, The equation $a^{M}=b^{N} c^{P}$ in a free group. Michigan Math. J. 9 (1962), 289-298.

WESLEYAN UNIVERSITY

\section{CONDITIONAL INTEGRABILITY OVER MEASURE SPACES}

\author{
BY HUBERT HALKIN
}

\section{Communicated by P. R. Halmos, March 3, 1965}

Introduction. Let $E^{n}$ be the $n$-dimensional Euclidean space, $(A, a, \mu)$ be a measure space and $f$ be a measurable function from $A$ into $E^{n}$. According to the theory of integration the function $f$ is either integrable or not integrable. The aim of this note is to show that nonintegrable functions can be divided into two classes: the totally unintegrable functions and the conditionally integrable functions. Moreover we shall show that the conditionally integrable functions may be further characterized by an index of conditional integration which can take the values $1,2, \cdots, n$.

Notations. A subset $\mathscr{N}$ of $\mathcal{Q}$ is a nest if for any $N_{1}$ and $N_{2} \in \Re$ we have either $N_{1} \subset N_{2}$ or $N_{2} \subset N_{1}$. A nest $\mathscr{T}$ of $a$ is called a sweeping nest for the measure space $(A, Q, \mu)$ if for each set $D \in Q$ with $\mu(D)<\infty$ and for each $\epsilon>0$ there exists a set $N \in \Re$ such that 